\title{
Memories of the Gold Foreign Exchange Market Based on a Moving $V$-Statistic and Wavelet-Based Multiresolution Analysis
}

\author{
Peng Zheng, ${ }^{1,2}$ Bin Liu $\mathbb{D}^{1,2,3}$ and Zhongli Zhou ${ }^{1,2}$ \\ ${ }^{1}$ College of Management Science, Chengdu University of Technology, Chengdu, Sichuan Province 610059, China \\ ${ }^{2}$ Geomathematics Key Laboratory of Sichuan Province, Chengdu, Sichuan Province 610059, China \\ ${ }^{3}$ School of Economics and Management, University of Electronic Science and Technology, Chengdu, Sichuan Province 611731, China \\ Correspondence should be addressed to Bin Liu; liubincim@163.com
}

Received 19 January 2018; Revised 18 July 2018; Accepted 7 August 2018; Published 23 August 2018

Academic Editor: Francisco R. Villatoro

Copyright (c) 2018 Peng Zheng et al. This is an open access article distributed under the Creative Commons Attribution License, which permits unrestricted use, distribution, and reproduction in any medium, provided the original work is properly cited.

\begin{abstract}
Memory in finance is the foundation of a well-established forecasting model, and new financial theory research shows that the stochastic memory model depends on different time windows. To accurately identify the multivariate long memory model in the financial market, this paper proposes the concept of a moving $V$-statistic on the basis of a modified $R / S$ method to determine whether the time series has a long-range dependence and subsequently to apply wavelet-based multiresolution analysis to study the multifractality of the financial time series to determine the initial data windows. Finally, we check the moving $V$-statistic estimation in wavelet analysis in the same condition; the paper selects the volatilities of the gold foreign exchange rates to evaluate the moving $V$-statistic. According to the results, the method of testing memory established in this paper can identify the breakpoint of the memories effectively. Furthermore, this method can provide support for forecasting returns in the financial market.
\end{abstract}

\section{Introduction}

The empirical analysis of long-term memory originated in the natural sciences. Since the 1980s, econometrists have introduced the long-term memory model in the financial field and considered that the cornerstones of the long-term memory of the finance market include the theories of noise trading [1], behavioral financial theory [2], and the fractal market hypothesis [3]. As the basis of an established forecasting model, many scholars have conducted extensive and indepth research and have formed the Hurst index technology based on time domain analysis [4-6] and the fractional order difference parameter technique based on frequency domain analysis [7-9]. There is substantial literature improving the ability of the accurate judgment for the long-term memory by optimizing parameter algorithms [10-12].

With the continuous improvement of traditional methods of estimation and inspection, scholars have applied the longterm memory model in the field of gold foreign exchange market. Bentes analyzes the robustness and consistency of long memory volatility of gold price returns during different crisis periods by FIGARCH model [13]. Ali Habibnia establishes the model for the world gold price with Logistic Smooth Transition Autoregressive (STAR) model concerning long memory effect and compared it with other models [14]. Yang $\mathrm{Na}$ explores the long memory property on gold price volatility by calculating the Hurst index and establishes a family of long memory forecasting models based on fractal analysis. It shows the inherent volatility quality of gold price sequences and it has strong predictive capabilities [15]. Maurice Omane-Adjepong examines the presence of longrange dependence in the world's gold market returns and volatility by using sampled historical daily gold market data to be less risky for hedging and portfolio diversification [16].

Mandelbrot (1997) introduced multifractal models to address the shortcomings of traditional models, which are not compatible with the stylized facts of time series, such as longterm memory and fat-tails in volatilities. Long-term memory models using wavelet-based multiresolution and Hurst index 
are widely researched from the perspective of multifractal in financial time series. As a comparatively new and powerful mathematical tool for time series analysis, multiresolution decomposition (MRD) is one of the basic tools of wavelet theory. Wavelet analysis is a time-frequency analysis method regarding signal in the time and frequency domain which has the ability of denoting partial signal characteristics. In 1989, Mallat and Meyer proposed the multiresolution analysis (MRA) theory and provided a numerical algorithm of discrete wavelet, namely, the Mallat tower algorithm (MTA) [17]. Wavelet-based multiresolution has a very wide range of applications in the financial sector, from descriptive analysis on different time scales to parameter estimation of multifractal properties and revelation of multifractality in crosscorrelativity. For example, the correlation function of the wave logarithm in different time scales is analyzed to reveal causal information from low frequencies to high frequencies [18]. Schmitt shows the multifractal characteristics of foreign exchange earnings and estimates parameters expressing the small and medium strength fluctuation characteristics under the general multiface structure by means of multifractal analysis regarding the five daily foreign exchange rates [19]. The multifractal property is proved to exist in the crosscorrelativity on the basis of an $\mathrm{RMB} /$ dollar exchange rate and daily price data of the Shanghai Composite Index [20].

The abovementioned literature studies the characteristics of memory by wavelet-based multiresolution analysis from the perspective of multifractal property depending on the fixed financial time series. Financial time series exhibit high degrees of nonlinear variability and multivariate longterm memory originates because of multiplicative interactions in different time windows; the multifractality of times series determines the multivariate long-term memory model.

To identify the breakpoint of memory in financial time series at a certain time scale and specific style of the multifractal form, this paper will estimate the dynamic value based on the specific branch level memory of the multifractal properties perspective. The remainder of the paper is organized as follows: after Section 1 outlines the development and application of long-term memory in financial time series, Section 2 introduces the concept of the moving $V_{n}(t, s)$ statistic after reviewing the modified $R / S$ theory and reviews wavelet-based multiresolution analysis, and Section 3 applies the model to evaluate the memory by selecting the highfrequency data of the gold price. The study's conclusions are presented in Section 4.

\section{The Moving $V_{n}(t, s)$-Statistic and Wavelet-Based Multiresolution Analysis}

2.1. Modified R/S Theory and V-Statistic. In 1991, Lo put forward the modified $R / S$ theory [22] based on the classic $R / S$ theory to better distinguish between long- and shortrange dependence. For the time series $\left\{x_{k}\right\}(k=1,2,3, \ldots, n)$, given a sample of observation, $x_{1}, x_{2}, x_{3}, \ldots, x_{n}$, the definition of the modified rescaled range theory is as follows. $Q_{n}(q)$ is the square root of a consistent estimator of the partial sum's variance.

$$
\begin{aligned}
& Q_{n}(q) \\
& \quad=\frac{1}{\widehat{\sigma}_{n}(q)}\left\{\max _{1 \leq k \leq n} \sum_{j=1}^{k}\left(x_{j}-\bar{x}_{n}\right)-\min _{1 \leq k \leq n} \sum_{j=1}^{k}\left(x_{j}-\bar{x}_{n}\right)\right\}
\end{aligned}
$$

where $\bar{x}_{n}=(1 / n) \sum_{j=1}^{n} x_{j}$ and $\bar{x}_{n}$ denotes the mean value of the time series. $\widehat{\sigma}_{n}(q)$ denotes the standard deviation of the time series after modification. This deviation involves not only sums of squared deviations of $x_{j}$, but also its weight of autocovariances up to lag $q$.

$$
\begin{aligned}
\widehat{\sigma}_{n}^{2}(q)= & \frac{1}{n} \sum_{j=1}^{n}\left(x_{j}-\bar{x}_{n}\right)^{2} \\
& +\frac{2}{n} \sum_{j=1}^{q} \omega_{j}(q)\left\{\sum_{i=j+1}^{n}\left(x_{i}-\bar{x}_{n}\right)\left(x_{i-j}-\bar{x}_{n}\right)\right\} \\
\omega_{j}(q)= & 1-\frac{j}{q+1}, \quad q<n,
\end{aligned}
$$

where $q$ denotes the lag factor of the time series, according to Andrews' (1991) data-dependent rule as in Lo (1991).

$$
q=\left[q^{*}\right]
$$

where $q^{*}=(2 n / 3)^{1 / 3} \cdot\left(\hat{\rho} /\left(1-\hat{\rho}^{2}\right)\right)^{2 / 3},\left[q^{*}\right]$ denotes the greatest integer less than or equal to $q^{*}$, and $\hat{\rho}$ is the estimated firstorder autocorrelation coefficient of the data.

The normalized classical Hurst-Mandelbrot rescaled range $V_{n}(q)$ :

$$
V_{n}(q)=\frac{Q_{n}(q)}{\sqrt{n}}
$$

Compared with the classical $R / S$ analysis method, the main advantage of the modified $R / S$ analysis method is to avoid computing the Hurst index. The standard deviation is modified by introducing the lag factor to exclude the short-term memory of the time series for testing long-term memory, which makes long-term memory detection more robust.

2.2. Definition of the Moving $V_{n}(t, s)$-Statistic. The characteristics of memory diversification are produced because the value of $V(q)$ differs based on different time windows selected under the multifractal properties of the financial time series conditions. This eliminates interference of the memory test from the initial time window, which contributes to the multifractal property, to observe the dynamic change process of memory in the financial time series. This paper proposes the concept of a moving $V_{n}(t, s)$-statistic based on a nonfixed scale on the basis of the classical $V(q)$ statistic. 
Definition 1. Given time series $\left\{x_{k}\right\}(k=1,2,3, \ldots, n)$, where $\mathrm{k}$ is the length of the time series:

$$
V_{n}(t, s)=\frac{Q_{t+s}\left(q_{t+s}\right)}{\sqrt{t+s}} \quad s \leq n-t
$$

where $t$ denotes length of time series in the initial time windows, namely, in the time series $\left\{x_{k}\right\}(k=1,2,3, \ldots t, \ldots, n)$, the initial data from the time series consist of $x_{1}, x_{2}, x_{3, \ldots .} x_{t} . s$ denotes the moving $V_{n}(t, s)$-statistic progress step $(s \leq n-t)$, with implied calculation accuracy.

In the process of testing the memory of the nonfixed scale by utilizing the moving $V_{n}(t, s)$-statistic, suppose that the starting data of time series for analysis will be written as $x_{m}(1<m<n)$, then the breakpoint of memory property of the time series $x_{m}, x_{m+1}, x_{m+2}, \ldots, x_{n}$ should be identified. The initial window data should satisfy the following conditions:

(i) As to the initial time window data, $x_{m-t}, x_{m-t+1}$, $x_{m-t+2}, \ldots, x_{m-1}$, under the condition of multilevel fractal analysis, the fractal level of samples $x_{m-t}, x_{m-t+1}$, $x_{m-t+2}, \ldots, x_{m-1}$ is at a lower fractal level than the time series data $x_{m}, x_{m+1}, x_{m+2}, \ldots, x_{n}$.

(ii) Under the condition of multilevel fractal analysis, the level of the fractal of the sample data $x_{m-t-u}, x_{m-t-u+1}, \ldots$, $x_{m-t-1}(u=2,3,4 \ldots)$ is the same as the level of the sample data $x_{m-t}, x_{m-t+1}, x_{m-t+2}, \ldots, x_{m-1}$.

The moving $V_{n}(t, s)$-statistic can eliminate all short-term memory within the target time windows, as well as the initial time window data, and would not affect memory independence in the target time window data.

2.3. Wavelet-Based Multiresolution Analysis. A multiresolution analysis (MRA) or multiscale approximation (MSA) is the design method of most relevant to discrete wavelet transforms (DWT) and the justification for the algorithm of the fast wavelet transform (FWT). It was introduced in the theory of differential equations (the ironing method) and the pyramid methods of image processing by Stephane Mallat and Yves Meyer in 1988/89. Multiresolution signal decomposition and the reconstruction algorithm (and the fast algorithm of orthogonal wavelet transform), generally called the Mallat algorithm, include two key steps: decomposition and reconstruction.

\subsubsection{Decomposition}

$$
\begin{aligned}
S_{2^{j}}^{d} f_{n} & =\sum_{k} \tilde{h}(2 n-k) \cdot S_{2^{j-1}}^{d} f_{k} \\
D_{2^{j}} f_{n} & =\sum_{k} \tilde{g}(2 n-k) \cdot S_{2^{j-1}}^{d} f_{k}
\end{aligned}
$$

\subsubsection{Reconstruction}

$$
S_{2^{j-1}}^{d} f_{n}=\sum_{k} h(n-2 k) \cdot S_{2^{j}}^{d} f_{k}+\sum_{k} g(n-2 k) \cdot D_{2^{j}} f_{k}
$$

Mallat's algorithm is useful in representing the wavelet transform as a pyramid [23]. The base of the pyramid is the original data of high resolution, and the top is a low-resolution approximation, and the size and resolution will be reduced as the pyramid upper moves. Many studies establish a prediction model via multiresolution analysis method to forecast gold price volatility [24-26]. This paper will use wavelet-based multiresolution analysis to explore memory feature in gold price volatility in different time scales. The four criteria as followed are considered for selecting the mother wavelet adopted in this paper [27].

(i) Vanishing moments: the wavelet function should have a small enough vanishing moments to represent multifractality of the high-frequency data.

(ii) Cutoff frequencies: the wavelet should provide not sharp cutoff frequencies to magnify the adjacent resolution levels.

(iii) Orthonormal: the wavelet basis should be orthonormal.

(iv) The similarity of wavelet coefficients: for applications where the information lasts for a very short instant, wavelets with less number of coefficients are better choices.

There are several well-known families of orthogonal wavelets. An incomplete list includes Harr, Meyer family, Daubechies family, Coiflet family, and Symmlet family [28]. Prior studies $[29,30]$ show that gold has nonlinear multiresolution characteristic in different time scales. Daubechies wavelets are selected in this paper due to their outstanding performance in detecting waveform discontinuities for evaluating the memorial breakpoint [31].

The hour returns of exchange rate between gold and the US dollar are chosen as the target data for detecting the memorial breakpoint of the high-frequency data from the perspective of multifractality, rather than not smoothing signal, while the larger the vanishing moment of wavelet filter is, the shaper its cutoff frequency is. So filter banks of Daubechies 3 (db3) are selected for determining the initial data window and evaluation in comparison with Daubechies $5(\mathrm{db} 5)$.

2.4. Analysis Process of the Moving $V_{n}(t, s)$-Statistic. To resolve multifractality of the time series, this paper utilizes wavelet analysis to recognize and reconstruct financial time series and later calculate the moving $V_{n}(t, s)$-statistic to determine the breakpoint of the memory. The results are then subjected to wavelet analysis for evaluation and this process is shown in Figure 1.

\section{Model Application in Gold Price Returns}

3.1. Data Sources and Descriptive Statistical Analysis. The hourly returns of exchange rate between gold and the US dollar (from MT4) from May 10, 2016, to December 16, 2016, create a total of 3630 observations. The exchange rate is quoted as the price of a dollar in terms of gold. The descriptive statistics are shown in Table 1, and returns for XAU/USD are shown in Figure 2. 


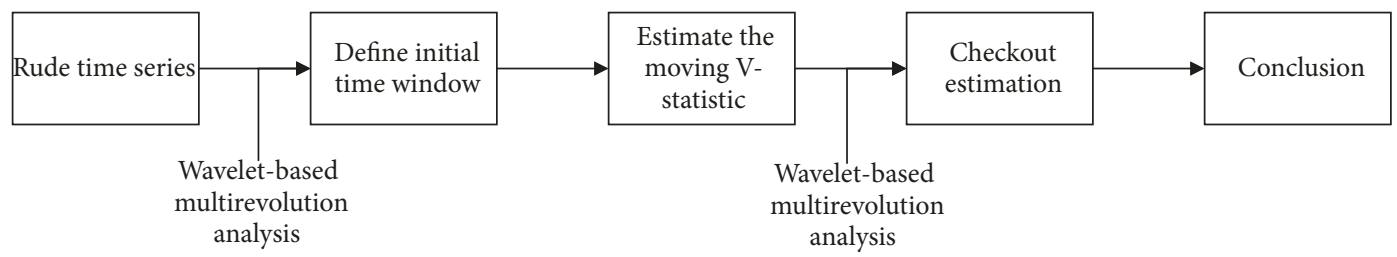

FIGURE 1: Moving $V_{n}(t, s)$-statistic analysis process.

TABLE 1: Descriptive statistic for volatilities of XAU/USD,

\begin{tabular}{lccccrr}
\hline Time range & Min & Max & Mean & Std & Skew & Kurt \\
\hline 2016.5.10 00:00- & 1129.36 & 1375.04 & \multirow{2}{*}{1285.28} & \multirow{2}{*}{55.61} & -0.62 & 2.48 \\
$2016.12 .1600: 00$ & & & & \\
\hline
\end{tabular}

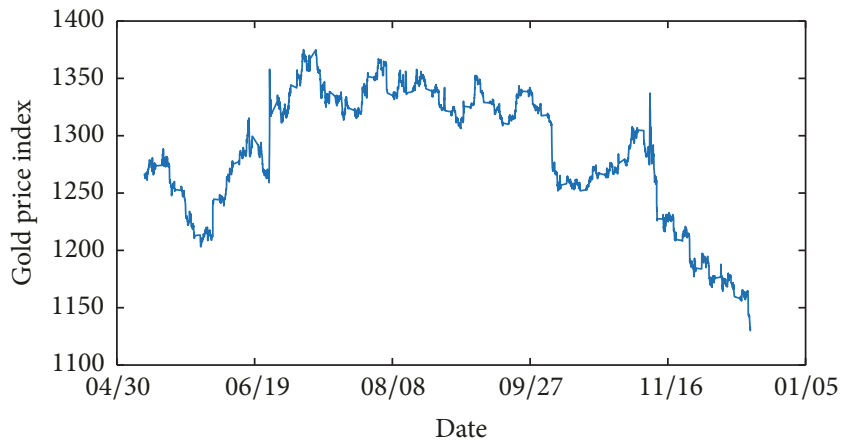

FIGURE 2: Return charts of XAU/USD.

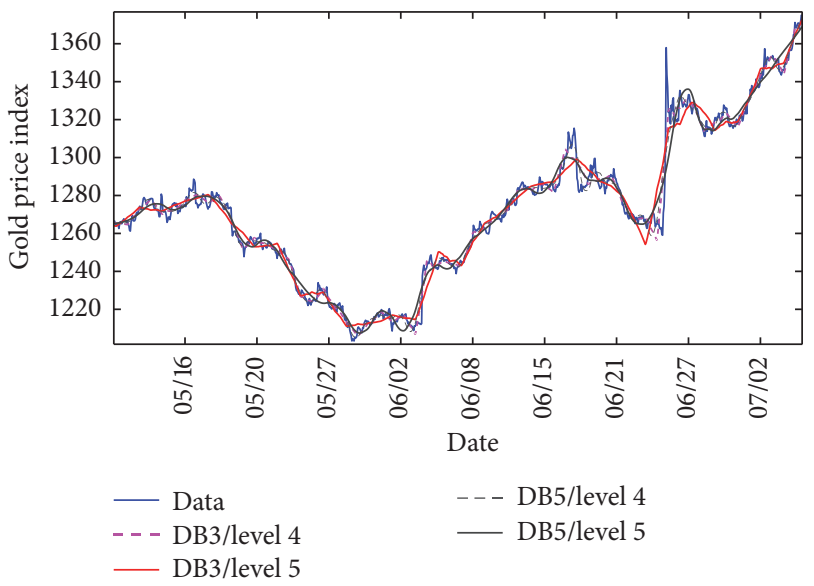

Figure 3: DB3 and DB5 wavelet analysis on Level 4/5.

3.2. The Initial Data Window Determination. The 622 observations, ranging from May 30, 2016, 15:00, to June 6, 2016, 19:00, are selected as the target window for calculating the moving $V_{n}(t, s)$-statistic. Before using the moving $V_{n}(t, s)$ statistic estimation to test the breakpoint of memory in the target window, the initial data window should be determined by wavelet-based multiresolution analysis. The analysis results are shown in Figures 3 and 4.

The lower fractal signals are filtered with synchronization after step-by-step analysis of the volatilities of XAU/USD,

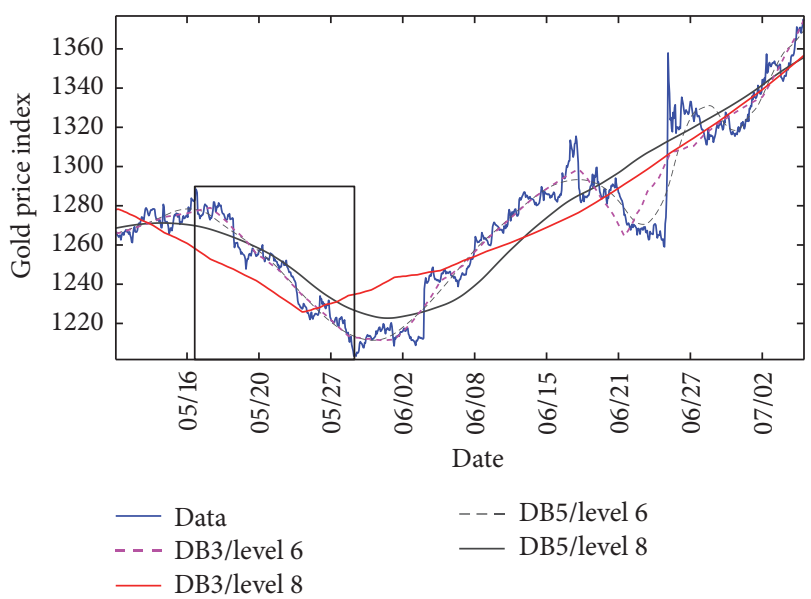

Figure 4: DB3 and DB5 wavelet analysis on Level 6/8.

ranging from May 16 to May 30 and the target data level after Level 4 and Level 5 analysis. As shown in Figure 3, after Level 6 analysis, the returns from May 16 to May 30 and the target window data are synchronously filtered completely and are the homogeneous fractal property after Level 8 resolution analysis. Both the results of wavelet analysis by DB3 and DB5 have the consistency in synchronous filter of the high frequency, while the reconstruction of the DB3 is sharper than DB5. This can be concluded as a result under the DB3 wavelet analysis conditions. The fractal property of the returns of XAU/USD from May 16 at 20:00 to May 30 at 15:00 is a lower fractal than the target window data, and it is the same level fractal as the volatilities before May 16. This finding meets the conditions that the returns can be treated as the initial window data. The length of the volatilities of $\mathrm{XAU} / \mathrm{USD}$ is 225 .

3.3. Estimation of the Moving $V_{n}(t, s)$-Statistic. To precisely evaluate the moving $V_{n}(t, s)$-statistic value for hourly returns, step 1 is used as the account step to analyze the data, $t=$ $225, s=1$. The estimation of moving $V_{n}(t, s)$-statistic is shown in Figure 5.

Under the null hypothesis of short-range dependence conditions, the moving $V_{n}(t, s)$-statistic gradually distributes 
TABLE 2: Moving $V_{n}(t, s)$-statistic under different level of the critical value [21].

\begin{tabular}{lllllllll}
\hline$P(V<x)$ & 0.005 & 0.025 & 0.050 & 0.100 & 0.200 & 0.300 & 0.400 & 0.500 \\
\hline$x$ & 0.721 & 0.809 & 0.861 & 0.927 & 1.018 & 1.090 & 1.157 & 1.223 \\
\hline$P(V<x)$ & 0.600 & 0.700 & 0.800 & 0.900 & 0.950 & 0.975 & 0.990 & 0.995 \\
\hline$x$ & 1.294 & 1.374 & 1.473 & 1.620 & 1.747 & 1.862 & 2.001 & 2.098 \\
\hline
\end{tabular}

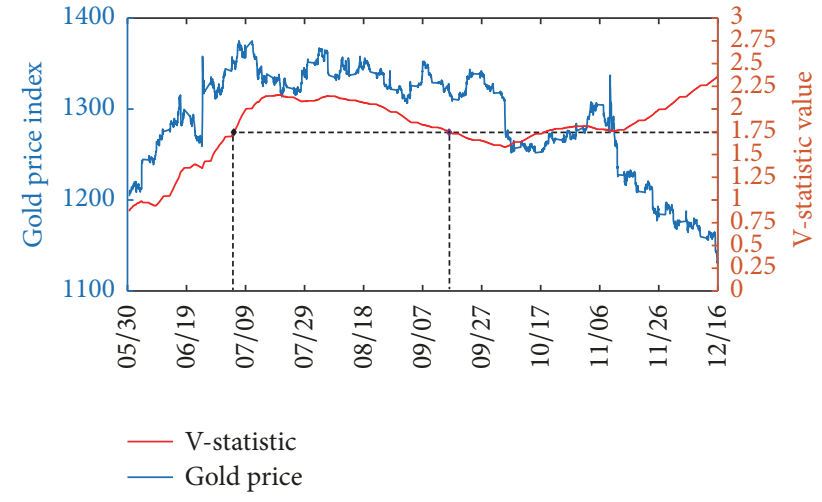

FIGURE 5: Moving $V_{n}(t, s)$-statistic evaluated for the volatilities of $\mathrm{XAU} / \mathrm{USD}$.

as the first-order brown bridge, and moving $V_{n}(t, s)$-statistic distributes as shown in Table 2.

The fluctuation of the moving $V_{n}(t, s)$-statistic is evaluated as shown in Figure 5; the moving $V_{n}(t, s)$-statistic value is greater than 1.747 for the first time until the target window data approach June 4 at 23:00. This finding suggests that with the $95 \%$ confidence level, the gold price index from May 30 at 15:00 to June 6 at 19:00 has the memory property at the first time based on the fractal level of the target window data. As time goes by, the peak value reaches 2.15 , which is merged around June 20. This finding indicates that the memory is most robust at the span of the target window data, which is when the volatilities of XAU/USD are near the peak value. The consistency of change between the moving $V_{n}(t, s)$ statistic estimation and the returns is shown in Figure 5. When time approaches September 16 at 9:00, the moving $V_{n}(t, s)$-statistic drops to less than 1.747. Under the condition of $95 \%$ confidence level, this indicates that the target window data memory disappears, which is the breakpoint on the same level of fractal memory.

3.4. Wavelet Analysis for Evaluation. To verify that there is a breakpoint in the memory properties in the target window data under the same condition, DB3 wavelet basis is also used as a wavelet base for wavelet analysis in order to evaluate breakpoint effectiveness. Wavelet-based multiresolution analysis is shown in Figure 6.

From Figure 6 through Levels 7 and 8 of multifractal analysis by DB3 and DB5, the volatilities of XAU/USD from June 6 at 19:00 to September 16 at 9:00 form a higher fractal level than the target window after the volatilities of XAU/USD reduce on September 16. This finding indicates that memory has turned, and the memory breakpoint in reconstructed

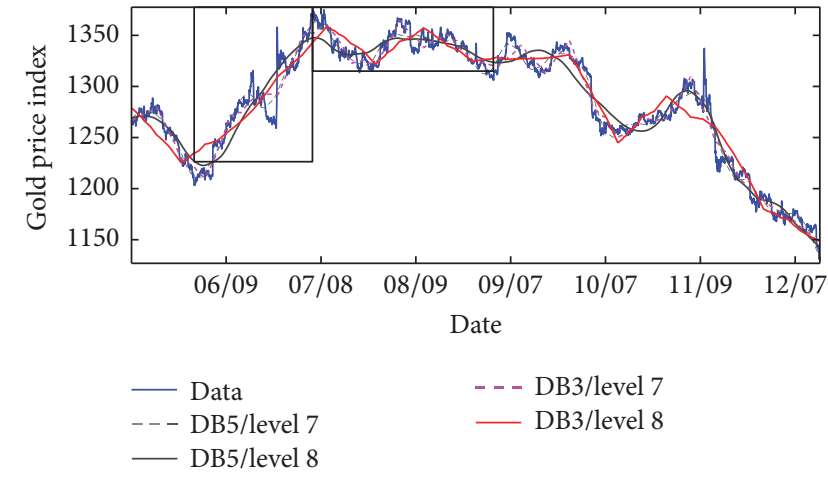

FIgURE 6: Moving $V_{n}(t, s)$-statistic checked out by wavelet analysis.

signal of the DB3 on Level 7 is more obvious and precious compared with DB5. The results based on wavelet-based multiresolution analysis and the calculation results of the moving $V_{n}(t, s)$-statistic remain the same.

\section{Conclusions}

There are important theoretical and practical implications of memory research in financial markets. The existence of memory under multifractal financial time series conditions and the interaction mechanism help to elucidate the multiple microstructure of the market, price behavior, and the management of financial markets.

This paper first reviews the principle of the memory test for the modified $R / S$ and subsequently proposes the definition of moving $V_{n}(t, s)$-statistic, which is the memory research index of specific hierarchical fractal after multifractal analysis. Finally, we have an example verification after wavelet-based multiresolution analysis together with hour high-frequency returns of XAU/USD from MT4. The validated results show that the moving $V_{n}(t, s)$-statistic based on wavelet-based multiresolution analysis effectively identifies the breakpoint of the memory of the specific multifractal level after decomposing it through wavelet analysis. The moving $V_{n}(t, s)$-statistic method can eliminate multivariate memory from the different window selected. This method makes the study of memory more specific, as well as providing the basic principle of the data window selected for building a forecasting model to eliminate effects from the memory from the selected window and to improve the model.

This paper utilizes wavelet-based multiresolution analysis to examine the multifractality of financial markets and to decompose and reconstruct with DB3 wavelet basis and DB5 wavelet basis. Actual result shows that DB3 wavelet basis is more appropriate than DB5. But the actual financial 
time series data are specific, complex, and multiply selfsimilar; Empirical Mode Decomposition (EMD), which has self-adaptability with target data, may be utilized to more accurately analyze the breakpoint of the memory for specific frequency signal.

\section{Data Availability}

The data used to support the findings of this study are available from the corresponding author upon request.

\section{Conflicts of Interest}

The authors declare no conflicts of interest.

\section{Acknowledgments}

This study was supported by National Key R\&D Program of China (2017YFC0601505); Opening Fund of Geomathematics Key Laboratory of Sichuan Province (Project no. scsxdz201601); Scientific Research Fund of Sichuan Province Education Department (Project no. 18ZB0062).

\section{References}

[1] R. Bloomfield, M. O’Hara, and G. Saar, "How noise trading affects markets: An experimental analysis," Review of Financial Studies, vol. 22, no. 6, pp. 2275-2302, 2009.

[2] J. Henrich, S. J. Heine, and A. Norenzayan, "The weirdest people in the world?" Behavioral and Brain Sciences, vol. 33, no. 2-3, pp. 61-83, 2010.

[3] M. Li, "Fractal time series-a tutorial review," Mathematical Problems in Engineering, vol. 2010, Article ID 157264, 26 pages, 2010.

[4] B. Mandelbrot, "New methods in statistical economics," Journal of Political Economy, vol. 71, no. 5, pp. 421-440, 1963.

[5] A. Carbone, G. Castelli, and H. E. Stanley, "Time-dependent Hurst exponent in financial time series," Physica A: Statistical Mechanics and Its Applications, vol. 344, no. 1-2, pp. 267-271, 2004.

[6] J. Alvarez-Ramirez, J. Alvarez, E. Rodriguez, and G. FernandezAnaya, "Time-varying Hurst exponent for US stock markets," Physica A: Statistical Mechanics and its Applications, vol. 387, no. 24, pp. 6159-6169, 2008.

[7] J. Geweke and S. Porter-Hudak, "The estimation and application of long-term memory time series models," Journal of Time Series Analysis, vol. 4, no. 4, pp. 221-238, 1983.

[8] B. Lashermes, S. Jaffard, and P. Abry, "Wavelet leader based multifractal analysis," in Proceedings of the 2005 IEEE International Conference on Acoustics, Speech, and Signal Processing, ICASSP '05, vol. 4, pp. 161-164, Philadelphia, PA, USA, March 2005.

[9] E. A. F. Ihlen, "Introduction to multifractal detrended fluctuation analysis in Matlab," Frontiers in Physiology, vol. 3, article 141, 2012.

[10] L. Giraitis, P. Kokoszka, R. Leipus, and G. Teyssière, "Rescaled Variance and Related Tests for Long-term memory in volatilities and Levels," Journal of Econometrics, vol. 112, no. 2, pp. 265-294, 2003.

[11] W. W. S. Wei, Time Series Analysis, 2013.
[12] S. J. Koopman, M. Ooms, and M. A. Carnero, "Periodic Seasonal Reg-ARFIMA-GARCH Models for Daily Electricity Spot Prices," Journal of the American Statistical Association, vol. 102, no. 477, pp. 16-27, 2007.

[13] S. R. Bentes, "Long memory volatility of gold price returns: how strong is the evidence from distinct economic cycles?" Physica A: Statistical Mechanics and its Applications, vol. 443, pp. 149160, 2016.

[14] A. Habibnia, Forecasting the World Gold Price Using Optimized Neuro-Fuzzy with Genetic Algorithm (Ga-Anfis) and Smooth Transition Regression with Long Memory (Fi-Star) Modelling, 2010.

[15] Y. Na, "The long memory property detection and prediction on gold price volatility by fractal analysis," Journal of Applied Statistics and Management, 2013.

[16] M. Omane-Adjepong and G. Boako, "Long-range dependence in returns and volatility of global gold market amid financial crises," Physica A: Statistical Mechanics and its Applications, vol. 472, pp. 188-202, 2017.

[17] S. G. Mallat, "A theory for multiresolution signal decomposition: the wavelet representation," IEEE Transactions on Pattern Analysis and Machine Intelligence, vol. 11, no. 7, pp. 674-693, 1989.

[18] A. Arnéodo, J. Muzy, and D. Sornette, "'Direct” causal cascade in the stock market," The European Physical Journal B, vol. 2, no. 2, pp. 277-282, 1998.

[19] F. Schmitt, D. Schertzer, and S. Lovejoy, "Multifractal analysis of foreign exchange data," Applied Stochastic Models and Data Analysis, vol. 15, no. 1, pp. 29-53, 1999.

[20] G. Cao, L. Xu, and J. Cao, "Multifractal detrended crosscorrelations between the Chinese exchange market and stock market," Physica A: Statistical Mechanics and its Applications, vol. 391, no. 20, pp. 4855-4866, 2012.

[21] F.-X. Huang, J.-D. Jin, and Y.-X. Li, "Comparable Analysis of Long-Term Memory of EUR/USD Based on Non-Parametrical Statistics," Management Science and Engineering, vol. 4, no. 3, pp. 117-127, 2010.

[22] A. W. Lo, "Long-term memory in stock market prices," Econometrica, vol. 59, no. 5, pp. 1279-1313, 1991.

[23] N. Dhillon, Analysis of Wavelet Transform And Fast Wavelet Transform for Image Compression: Review, 2012.

[24] M. Fathian and A. N. Kia, "Exchange rate prediction with multilayer perceptron neural network using gold price as external factor," Management Science Letters, vol. 2, no. 2, pp. 561-570, 2012.

[25] N. L. Alvarez-Berríos and T. Mitchell Aide, "Global demand for gold is another threat for tropical forests," Environmental Research Letters, vol. 10, no. 1, article 14006, 2015.

[26] C. Ntantamis and J. Zhou, "Bull and bear markets in commodity prices and commodity stocks: Is there a relation?" Resources Policy, vol. 43, pp. 61-81, 2015.

[27] Z. Wang, S. McConnell, R. S. Balog, and J. Johnson, "Arc fault signal detection - Fourier transformation vs. wavelet decomposition techniques using synthesized data," in Proceedings of the 40th IEEE Photovoltaic Specialist Conference, PVSC 2014, pp. 3239-3244, June 2014.

[28] S. Mallat, A Wavelet Tour of Signal Processing, 1998.

[29] M. R. Rahimi, M. Sabounchi, and F. Hanzaleh, "Forecasting Gold Price with Hybrid Model," Asian Journal of Research in Banking and Finance, vol. 4, no. 8, pp. 53-64, 2014. 
[30] H. Mombeini and A. Yazdani-Chamzini, "Modeling gold price via artificial neural network," Journal of Economics, Business and Management, vol. 3, no. 7, pp. 699-703, 2015.

[31] L. Zhang and P. Bao, "Edge detection by scale multiplication in wavelet domain," Pattern Recognition Letters, vol. 23, no. 14, pp. 1771-1784, 2002. 


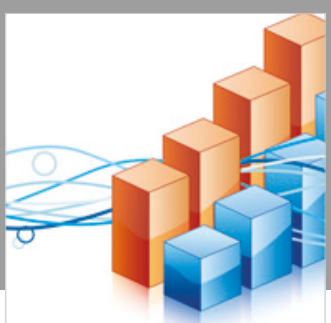

Advances in

Operations Research

\section{-n-m}
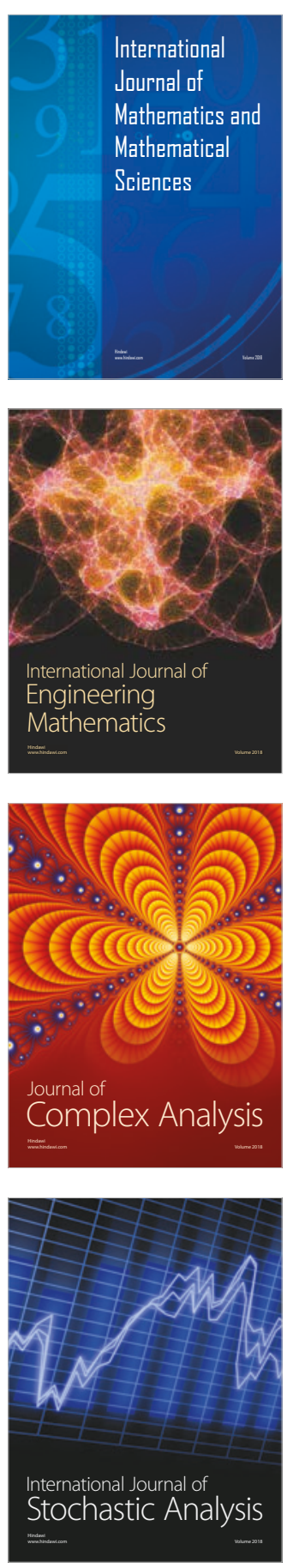
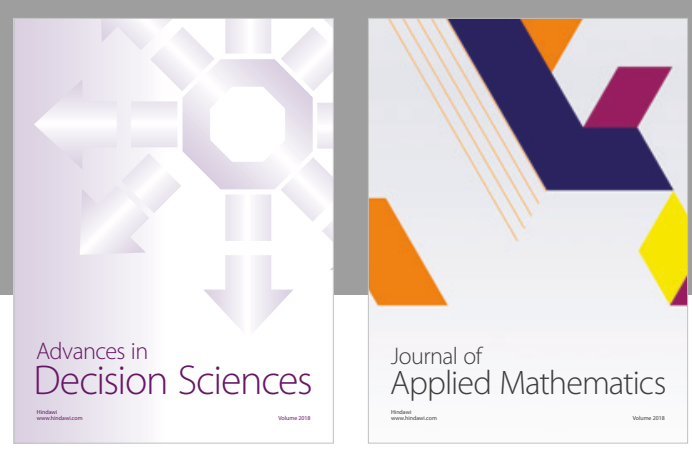

Journal of

Applied Mathematics
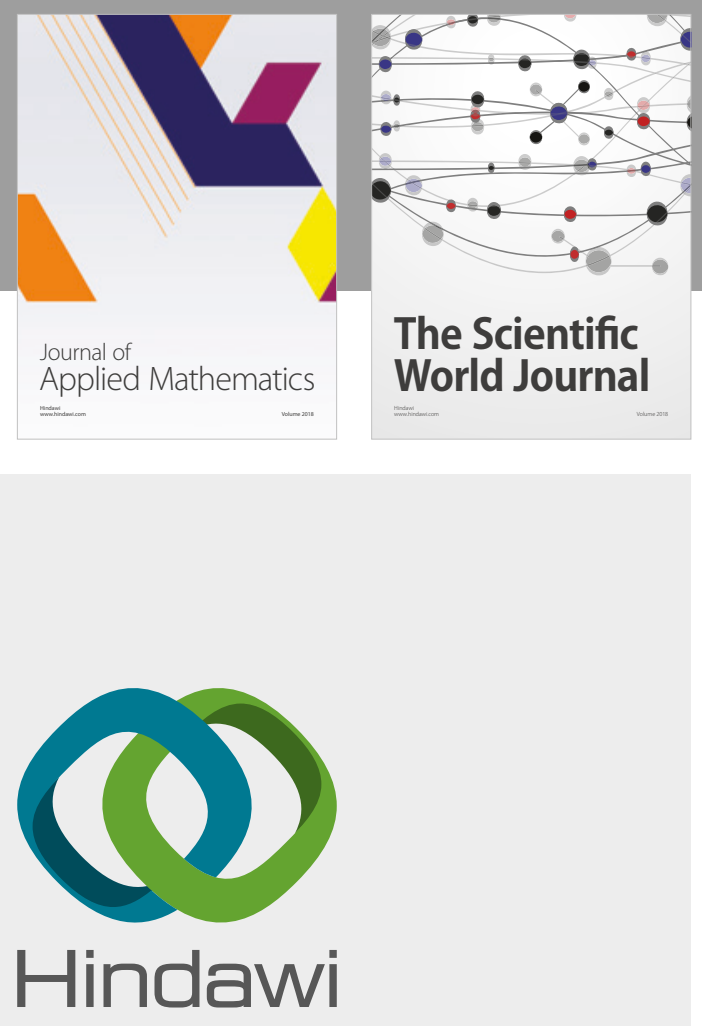

Submit your manuscripts at

www.hindawi.com

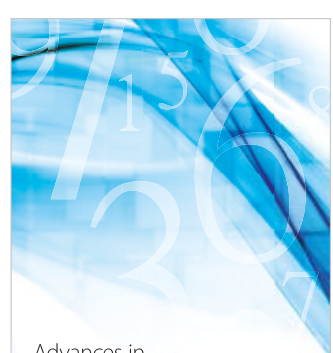

Advances in
Numerical Analysis
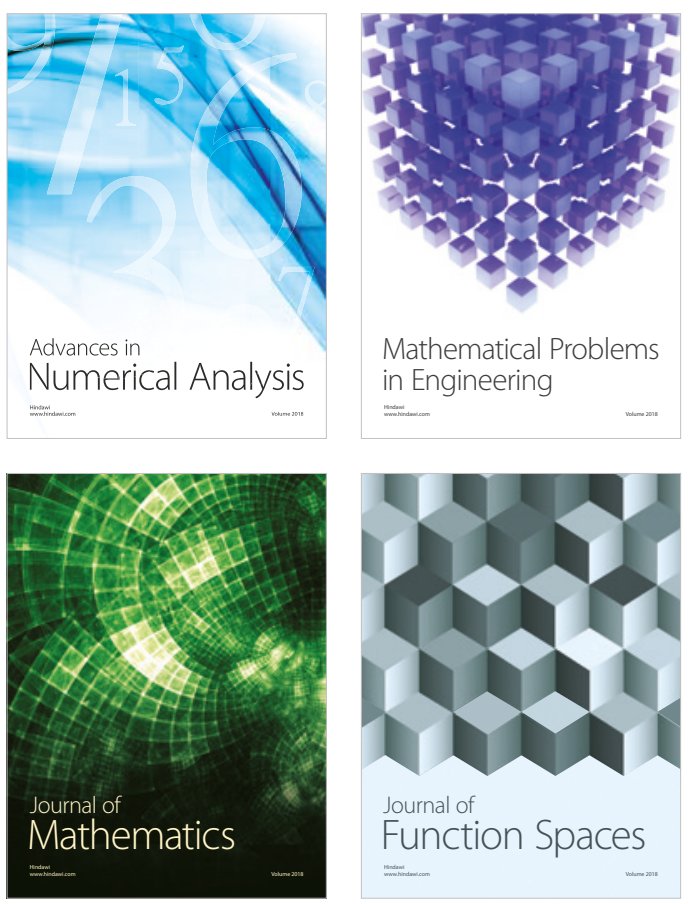

Mathematical Problems in Engineering

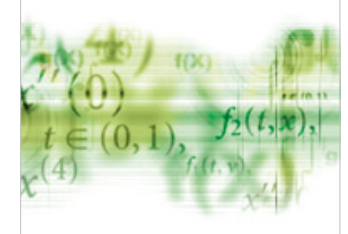

International Journal of

Differential Equations

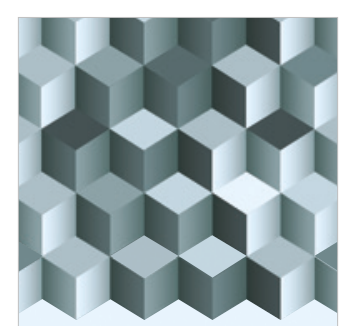

Journal of

Function Spaces

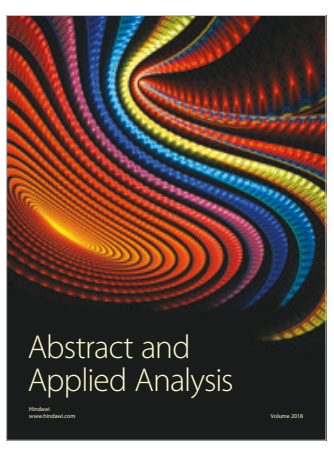

The Scientific

World Journal

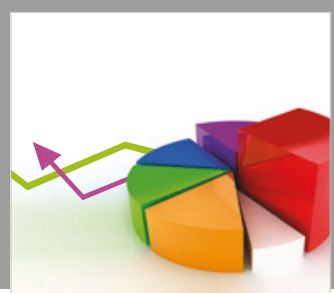

Journal of

Probability and Statistics
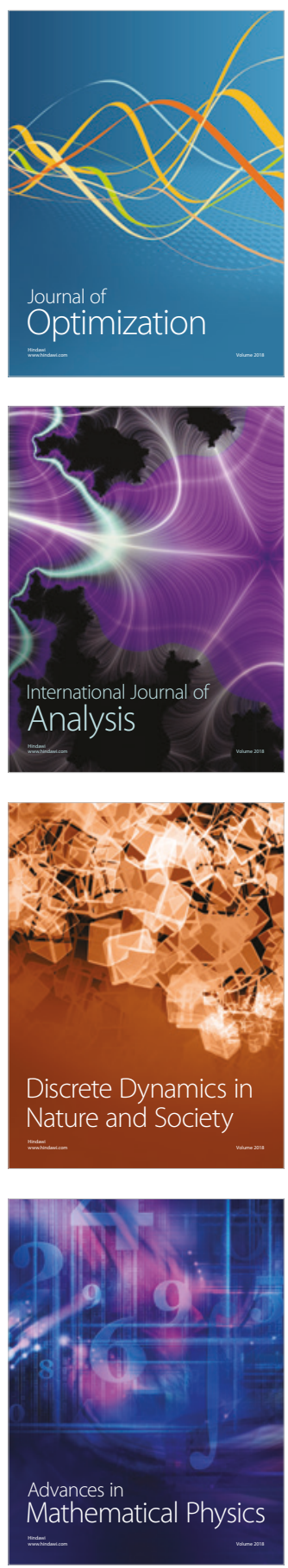